\title{
Elevated preoperative Galectin-3 is associated with acute kidney injury after cardiac surgery
}

\author{
Moritz Wyler von Ballmoos ${ }^{1}$, Donald S. Likosky ${ }^{2,3}$, Michael Rezaee ${ }^{4}$, Kevin Lobdell ${ }^{5}$, Shama Alam ${ }^{6}$, Devin Parker ${ }^{6}$, \\ Sherry Owens ${ }^{6}$, Heather Thiessen-Philbrook ${ }^{7}$, Todd MacKenzie ${ }^{6,8}$ and Jeremiah R. Brown ${ }^{6,8,9^{*}}$
}

\begin{abstract}
Background: Previous research suggests that novel biomarkers may be used to identify patients at increased risk of acute kidney injury following cardiac surgery. The purpose of this study was to evaluate the relationship between preoperative levels of circulating Galectin-3 (Gal-3) and acute kidney injury after cardiac surgery.

Methods: Preoperative serum Gal-3 was measured in 1498 patients who underwent coronary artery bypass graft (CABG) surgery and/or valve surgery as part of the Northern New England Biomarker Study between 2004 and 2007. Preoperative Gal-3 levels were measured using multiplex assays and grouped into terciles. Univariate and multinomial logistic regression was used to assess the predictive ability of Gal-3 terciles and AKI occurrence and severity.

Results: Before adjustment, patients in the highest tercile of Gal-3 had a 2.86-greater odds of developing postoperative KDIGO Stage 2 or $3(p<0.001)$ and 1.70-greater odds of developing KDIGO Stage $1(p=<0.001)$, compared to the first tercile. After adjustment, patients in the highest tercile had 2.95-greater odds of developing KDIGO Stage 2 or $3(p<0.001)$ and 1.71-increased odds of developing KDIGO Stage $1(p=0.001)$, compared to the first tercile. Compared to the base model, the addition of Gal-3 terciles improved discriminatory power compared to without Gal-3 terciles (test of equality $=0.042$ ).

Conclusion: Elevated preoperative Gal-3 levels significantly improves predictive ability over existing clinical models for postoperative AKI and may be used to augment risk information for patients at the highest risk of developing AKI and AKI severity after cardiac surgery.
\end{abstract}

Keywords: Acute kidney injury (AKI), Galectin-3 (Gal-3), Cardiac surgery, Prediction, Biomarkers

\section{Background}

Acute kidney injury (AKI) is a well recognized complication following cardiac surgery, and significantly affects morbidity and mortality $[1,2]$. Up to $40 \%$ of patients develop AKI after cardiac surgery and places patients at 5-fold higher risk of death during hospitalization [3]. AKI has also been associated with hospital readmissions following cardiac surgery and hospitalization for heart failure or acute myocardial infarction (MI) [4-8].

\footnotetext{
*Correspondence: jbrown@dartmouth.edu; jeremiah.r.brown@dartmouth.edu ${ }^{6}$ The Dartmouth Institute for Health Policy and Clinical Practice, Geisel School of Medicine, Lebanon, $\mathrm{NH}$, USA

${ }^{8}$ Department of Biomedical Data Science, HB 7505 Dartmouth-Hitchcock Medical Center, Lebanon, NH NH 03756, USA

Full list of author information is available at the end of the article
}

Conventional metrics used to define and monitor the progression of AKI, such as serum creatinine and blood urea nitrogen levels, are insensitive, nonspecific and change notably only after significant kidney injury [8]. Identifying patients at increased risk of AKI prior to surgery is critical to take preventative measures and counsel patients on potential outcomes after cardiac surgery. A timely diagnosis would allow for earlier clinical intervention, greater care management prior to surgery, improved patient engagement and could improve patient outcomes.

New biomarkers allow a diagnosis to be made earlier than conventional measures, and allows kidney injury to be diagnosed even in the absence of concurrent or subsequent dysfunction. Biomarkers have been utilized to

(c) The Author(s). 2018 Open Access This article is distributed under the terms of the Creative Commons Attribution 4.0 International License (http://creativecommons.org/licenses/by/4.0/), which permits unrestricted use, distribution, and 
investigate AKI and augment the prediction of AKI risk and other complications following cardiac surgery [912]. A specific and sensitive marker of AKI risk could improve risk stratification, potentially identify patients that will benefit from greater care management prior to surgery and alert clinicians to individuals that will need earlier interventions to prevent AKI. Current risk prediction models for AKI following cardiac surgery have been developed on patient and disease characteristics alone. The addition of a specific protein biomarker may improve predictive ability over existing clinical models and may augment risk information for patients at higher risk of AKI after surgery.

Galectin-3 (Gal-3) is a beta-galactoside-binding lectin that has emerged as a key regulator of inflammation and tissue fibrosis [13]. Experimental studies in models of cancer, congestive heart failure and inflammatory disease have demonstrated that Gal-3 expression is elevated in these pathologic conditions [9]. In animal models, Gal-3 is acutely up-regulated in the kidneys in response to ischemic and toxic injury and is associated with renal fibrosis [14-17]. In humans, elevated levels of circulating Gal-3 have been found to be associated with increased risk of incident chronic kidney disease (CKD) and loss of kidney function over time [11, 18]. Gal-3 therefore can be considered a marker of both acute and chronic inflammatory processes in the kidneys, even in the absence of traditional clinical markers of renal injury.

There is an urgent need to analyze the predictive utility of Gal-3 to identify patients at greater risk of developing postoperative AKI. To date, Gal-3 has not been investigated as a potential biomarker for AKI in humans. The purpose of this study was to evaluate the assocation between preoperative Gal-3 and postoperative AKI in a large cohort of cardiac surgical patients.

\section{Methods \\ NNE biomarker study}

This study expands on the experience of Northern New England Cardiovascular Disease Study Group (NNECDSG), a regional collaborative consortium founded in 1987. All eight hospitals in this consortium submit data on cases with validation of procedure numbers and mortality conducted every 2 years. The NNECDSG registry contains data on patient characteristics, procedural indications, clinical variables and in-hospital outcomes. Data are periodically validated to ensure that all procedures and endpoints included in the registry have been accurately assessed. The NNECDSG has extensive experience in risk prediction in CABG surgery $[4,5]$. The Northern New England (NNE) Biomarker Study is an initiative designed to assess the role of biomarkers in cardiac surgery.

Patient, procedural and outcome data were collected from patients undergoing coronary CABG surgery.
Those undergoing CABG incidental to heart valve repair or replacement, resection of a ventricular aneurysm, or other surgical procedure were not included. Only patients that had biomarker levels collected were retained in the final analyses $(n=1498)$. For the present study, the sample included patients undergoing emergent, urgent and non-urgent CABG surgeries. Investigators and patients were blinded to the collected biomarker levels. The Committee for the Protection of Human Subjects at Dartmouth College (Institutional Review Board) approved this study for both the prospective cohort with patient consent and the linkage of readmission and mortality events.

\section{Galectin-3}

Preoperative levels of Gal-3 was the main exposure of interest for this study. Blood samples were preoperatively collected prior to incision at each participating site in a $10-\mathrm{mL}$ serum tube. Preoperative biomarker levels were measured using custom made multiplex ELISA assays (Meso Scale Discovery, Rockville, MD). Blood was allowed to clot at room temperature for 20 min to separate out the red blood cells, the tubes were centrifuged at $3500 \mathrm{rpm}$ for $20 \mathrm{~min}$, and the sera stored at the respective medical centers below -80 degree Celcius until transportation on dry ice to the Laboratory for Clinical and Biomedical Research in Colchester, Vermont where they were stored at -80 degree Celcius until measurement. Frozen serum was analyzed at a central laboratory, at the same time for biomarker measurement. Biomarkers were linked to the NNECDSG cohort to conduct the preoperative risk prediction modeling. Biomarkers were evaluated as continuous variables, natural log-transformed, and as terciles.

\section{Acute kidney injury}

The primary outcome of this study was the development of AKI after cardiac surgery. The last serum creatinine (SCr) prior to cardiac surgery and highest postoperative $\mathrm{SCr}$ prior to discharge were used to classify the stage of AKI. AKI stages were defined by the Kidney Disease: Improving Global Outcomes (KDIGO) definition as follows [19]: Stage 1: increase in $\mathrm{SCr}$ by $\geq 0.3 \mathrm{mg} / \mathrm{dL}$ within $48 \mathrm{~h}$ or $\geq 1.5$ times baseline within 7 days; Stage 2: increase in $\mathrm{SCr}$ to 2 to 2.9-fold baseline; and Stage 3: increase in $\mathrm{SCr}$ to 3.0 times baseline or increase in $\mathrm{SCr}$ to $\geq 4.0 \mathrm{mg} /$ $\mathrm{dL}$ or initiation of renal replacement therapy. Due to the small proportion of patients in KDIGO stage 2 and 3, we bundled stage 2 or 3 patients' outcomes in this report.

\section{Statistical analysis}

We evaluated the Gal-3 measurements to determine the association with the primary outcome (AKI) using 
univariate and multinomial logistic regression. Postoperative outcomes were compared using chi-squared tests, and continuous data were compared using the ANOVA test with the Bonferroni correction. Adjustment was carried out using variables from the Society of Thoracic Surgeons (STS) readmission model (Appendix) [20].To evaluate the association of the biomarker with AKI outcomes, we divided the cohort into terciles on the basis of Gal-3 values, where the lowest tercile is the reference group. We included indicator variables for the middle and highest terciles. We applied the mean imputation replacement method to account for variables with missing values. All biomarker values below the assay's lower quantitative limit were assigned the lower limit of detection. The performance of the risk model was assessed by measuring the total area under the receiver operating characteristic curve (AUC or c-statistic). Standard errors and 95\% confidence intervals were estimated for the c-statistic using a bootstrap method. All analyses were conducted using Stata 13.1 College Station, TX).

\section{Secondary Analyes}

We compared the incremental value of Gal-3 to preoperative eGFR, a traditional risk marker of AKI. We adjusted the model using the STS readmission model and used the test of equality of ROC areas to assess differences in model performance. We calculated the Net Reclassification Improvement (NRI) and Integrated Discrimination Improvement (IDI) indices for risk models including Gal-3 and eGFR values.

Finally, we also created an alternative final model including both preoperative $\mathrm{N}$-terminal pro b-type natriuretic peptide (NT-proBNP) values and preoperative Gal-3, in addition to the base STS readmission prediction model, to assess the predictive power using a combination of biomarkers. We compared model performance using the test of equality of ROC areas.

\section{Results}

Overall, 1489 patients were included in the study. 481 (32.1\%) developed AKI within KDIGO Stage 1 (26.3\%) and 87 (5.8\%) experienced KDIGO Stage 2 or 3 (Fig. 1). Patient and procedural characteristics are summarized in Table 1. Patient and procedural characteristics and the association with Gal-3 tercile measurements are summarized in Table 2. Gal-3 sample measurements ranged from 1.38 to $102.35 \mathrm{ng} / \mathrm{mL}$ with a median (Q1, Q3) value of $10.30 \mathrm{ng} / \mathrm{mL}$ (6.96 to $14.67 \mathrm{ng} / \mathrm{mL}$ ).

Among patients studied, there was a significant difference between postoperative AKI incidence for older patients and patients with a higher BMI, history of smoking, atrial fibrillation, congrestive heart failure (CHF), diabetes, ejection fraction $<40$, prior MI, vascular disease, received transfused blood, and red blood cells transfused postoperatively. There is a significant relationship between elevated Gal-3 measurements and increased AKI severity $(p<0.001)$. For patients in the lowest tercile of Gal-3, 22.9\% experienced KDIGO Stage 1 compared to $31.6 \%$ in the highest biomarker tercile. Similarly, for patients in the highest Gal-3 tercile, $1.8 \%$ of patients experienced KDIGO Stage 3 compared to $1.2 \%$ in the lowest tercile.

Table 3 describes the unadjusted and adjusted results for the AKI risk model and Gal-3 biomarker terciles. Before adjustment, patients in the highest tercile of Gal-3 had a 2.86-greater odds of developing postoperative KDIGO Stage 2 or 3 AKI $(p<0.001)$ and 1.70-greater odds of developing KDIGO Stage $1(p<0.001)$. After adjustment, patients in the highest tercile had 2.95-greater odds of developing KDIGO Stage 2 or 3

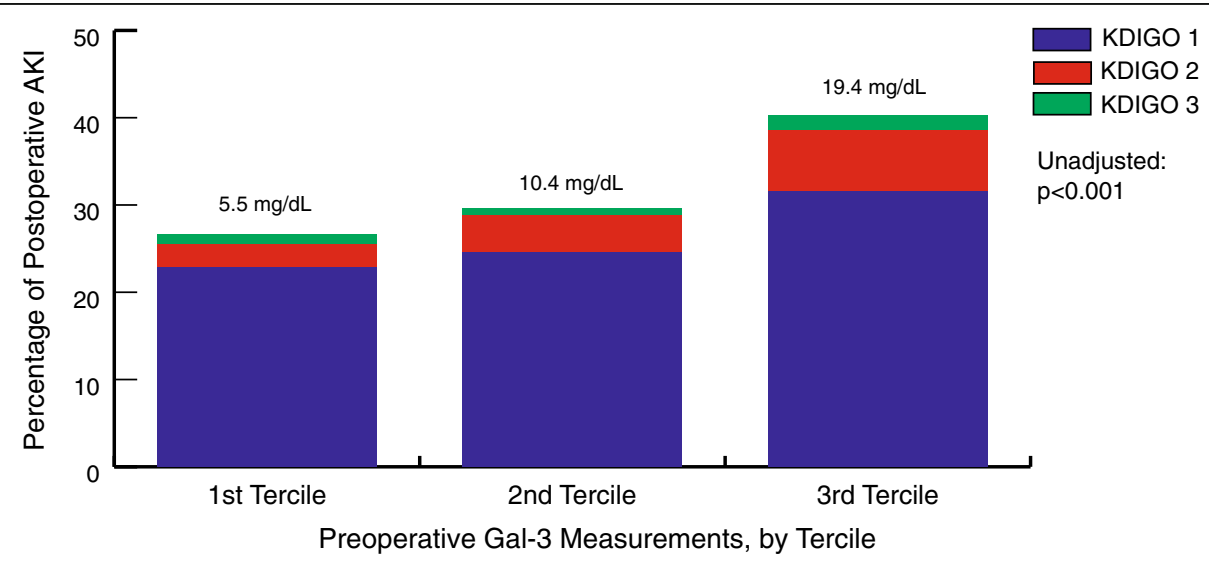

Fig. 1 Association of preoperative Galectin-3 and postoperative AKI severity after coronary artery bypass graft surgery, by Galectin-3 tercile. The mean Galectin-3 measurement for each tercile increases stepwise with postoperative AKI severity. There is a significant relationship between elevated preoperative Galectin-3 and AKI severity by KDIGO 
Table 1 Patient and procedural characteristics and postoperative AKI occurrence

\begin{tabular}{|c|c|c|c|}
\hline & $\begin{array}{l}\text { No AKI } \\
(N=1017)\end{array}$ & $\begin{array}{l}\text { AKI } \\
(N=481)\end{array}$ & $p$ value \\
\hline$\overline{\text { Age }^{*}}$ & $64.02 \pm 9.99$ & $68.08 \pm 9.90$ & 0.000 \\
\hline Female & $23.8 \%$ & $23.0 \%$ & 0.740 \\
\hline$\left.B M\right|^{*}$ & $29.22 \pm 5.17$ & $30.35 \pm 6.07$ & 0.000 \\
\hline $\mathrm{BSA}^{*}$ & $2.03 \pm 0.24$ & $2.04 \pm 0.26$ & 0.200 \\
\hline Smoker & $26.1 \%$ & $16.6 \%$ & 0.000 \\
\hline Atrial fibrillation & $5.2 \%$ & $9.3 \%$ & 0.003 \\
\hline $\mathrm{CHF}$ & $8.4 \%$ & $13.5 \%$ & 0.002 \\
\hline Pre-operative creatinine* & $1.08 \pm 1.02$ & $1.15 \pm 0.43$ & 0.108 \\
\hline Diabetes & $32.8 \%$ & $43.3 \%$ & 0.000 \\
\hline$E F<40$ & $8.9 \%$ & $14.0 \%$ & 0.003 \\
\hline Hypertension & $80.2 \%$ & $81.7 \%$ & 0.503 \\
\hline Pre-operative IABP & $3.2 \%$ & $5.7 \%$ & 0.019 \\
\hline Prior Ml & & & 0.004 \\
\hline None & $59.7 \%$ & $49.8 \%$ & \\
\hline$<24$ h preop & $1.5 \%$ & $1.9 \%$ & \\
\hline$>24 \mathrm{~h} \&<7$ days & $17.7 \%$ & $21.9 \%$ & \\
\hline$>7 \&<365$ days & $8.4 \%$ & $12.5 \%$ & \\
\hline$>365$ days & $12.7 \%$ & $13.9 \%$ & \\
\hline VAD & $24.5 \%$ & $32.5 \%$ & 0.001 \\
\hline Unstable angina & $55.3 \%$ & $56.0 \%$ & 0.780 \\
\hline COPD & $12.4 \%$ & $14.3 \%$ & 0.321 \\
\hline LM stenosis & $33.3 \%$ & $35.0 \%$ & 0.520 \\
\hline Prior CABG & $1.8 \%$ & $3.3 \%$ & 0.064 \\
\hline Prior $\mathrm{PCl}$ & $20.3 \%$ & $18.4 \%$ & 0.396 \\
\hline Priority & & & 0.297 \\
\hline Emergency or emergent salvage & $1.5 \%$ & $2.7 \%$ & \\
\hline Urgent & $67.6 \%$ & $67.9 \%$ & \\
\hline Non-urgent & $30.9 \%$ & $29.5 \%$ & \\
\hline Received transfused blood & $29.1 \%$ & $54.9 \%$ & 0.000 \\
\hline pRBCs transfused pre-operatively & & & 0.385 \\
\hline 0 & $98.5 \%$ & $97.3 \%$ & \\
\hline 1 & $0.4 \%$ & $1.0 \%$ & \\
\hline 2 & $0.7 \%$ & $1.3 \%$ & \\
\hline 3 or more & $0.4 \%$ & $0.4 \%$ & \\
\hline $\begin{array}{l}\text { pRBCs transfused } \\
\text { post-operatively }\end{array}$ & & & 0.000 \\
\hline 0 & $77.8 \%$ & $57.9 \%$ & \\
\hline 1 & $5.9 \%$ & $9.1 \%$ & \\
\hline 2 & $10.7 \%$ & $16.8 \%$ & \\
\hline 3 or more & $5.7 \%$ & $16.2 \%$ & \\
\hline Pump time (mean, SD) & $100.88 \pm 32.01$ & $112.44 \pm 37.43$ & 0.000 \\
\hline
\end{tabular}

$A K I$ acute kidney injury, $B M I$ body mass index $\left(\mathrm{kg} / \mathrm{m}^{2}\right), B S A$ body surface area $\left(\mathrm{m}^{2}\right), C A B G$ coronary artery bypass graft, $C H F$ congestive heart failure, $C O P D$ chronic obstructive pulmonary disease, $I A B P$ intra-operative balloon pump, $\mathrm{MI}$ myocardial infarction, $\mathrm{PCl}$ percutaneous coronary intervention, $R B C$ red blood cell, $S D$ standard deviation *signifies continuous variables $(p<0.001)$ and 1.71-increased odds of developing KDIGO Stage $1(p=0.001)$. When preoperative levels of NT-proBNP were added to the risk prediction model, we observed similar results. Patients in the highest tercile of Gal-3 had 2.85-greater odds of developing KDIGO Stage 2 or 3 AKI $(p=0.001)$ and patients in the lowest tercile at 1.65-greater odds of KDIGO Stage 1 AKI $(p=0.003)$.

The base and augmented models are summarized in Table 4. The base model yieled a c-statistic of $0.69(95 \%$ CI: $0.66-0.71)$. The base model with the addition of preoperative Gal-3 terciles yielded a c-statistic of $0.70(95 \%$ CI: $0.67-0.72$ ) and has a significant ROCCOMP $p$ value of 0.042 compared to the base model alone. With the addition of Gal-3 and NT-proBNP terciles to the base model, the c-statistic remains at 0.70 (95\% CI: 0.68 0.73) and is significantly improved from the base model alone $($ ROCCOMP $p$ value $=0.005)$.

In an exploratory analysis where we compared preoperative Gal-3 terciles to preoperative eGFR values, we did not find an appreciable difference between the two makers and risk of developing AKI. Models comparing preoperative Gal-3 tercile values to continuous eGFR values are reported in Table 5.

\section{Discussion}

We are the first to demonstrate a significant relationship between the inflammatory biomarker Gal-3 and AKI in a multi-site, prospectively enrolled cohort of patients undergoing cardiac surgery. We found Gal-3 concentrations increased concurrently with decreasing kidney function. In our study, patients in the highest tercile of preoperative Gal-3 levels had 1.7 times the adjusted odds of KDIGO Stage 1 AKI compared to patients in the lowest tercile of Gal-3. Patients in the highest tercile of preoperative Gal-3 also had 2.9 times the adjusted odds of KDIGO Stage 2 or 3 AKI compared to the lowest tercile.

Gal-3 is a well-established biomarker for cardiac fibrosis, ventricular dysfunction, and poor prognosis in heart failure [21-23]. In addition, Gal-3 has demonstrated diagnostic and prognostic value in diseases of the kidney $[9,11,18]$. Drechsler et al. found a positive association between elevated levels of Gal-3 and adverse outcomes in patients with preexisting renal disease. Additionally, in the well recognized Framingham Heart Study, researchers demonstrated that elevated Gal-3 levels precede the development of CKD [10, 11]. Prior to our analysis, studies examining the relationship between Gal-3 and acute renal injury after surgery had been limited to animal models. Multiple animal studies have demonstrated that Gal-3 expression is up-regulated in the kidneys in response to ischemic and toxic injury and is associated with renal fibrosis [14-17]. 
Table 2 Patient and procedural characteristics and association with Gal-3 terciles

\begin{tabular}{|c|c|c|c|c|c|}
\hline Patient characteristics & Overall & 1st Tercile & 2nd Tercile & 3rd Tercile & $p$ value \\
\hline \multicolumn{6}{|l|}{ KDIGO } \\
\hline No AKI & $67.9 \%$ & $73.4 \%$ & $70.5 \%$ & $59.6 \%$ & \multirow[t]{4}{*}{$<0.001$} \\
\hline Stage 1 & $26.3 \%$ & $22.9 \%$ & $24.6 \%$ & $31.6 \%$ & \\
\hline Stage 2 & $4.5 \%$ & $2.6 \%$ & $4.2 \%$ & $6.9 \%$ & \\
\hline Stage 3 & $1.3 \%$ & $1.2 \%$ & $0.8 \%$ & $1.8 \%$ & \\
\hline $\mathrm{Age}^{\mathrm{a}}$ & $65.7 \pm 9.9$ & $63.6 \pm 10.0$ & $65.5 \pm 9.6$ & $66.8 \pm 10.6$ & 0.464 \\
\hline Female & $22.7 \%$ & $18.2 \%$ & $20.6 \%$ & $32.0 \%$ & $<0.001$ \\
\hline$B M l^{\mathrm{a}}$ & $29.6 \pm 5.5$ & $29.6 \pm 5.2$ & $29.6 \pm 5.5$ & $29.8 \pm 5.9$ & 0.464 \\
\hline$B S A^{a}$ & $2.0 \pm 0.2$ & $2.0 \pm 0.3$ & $2.1 \pm 0.2$ & $2.0 \pm 0.3$ & 0.464 \\
\hline Smoker & $21.4 \%$ & $24.4 \%$ & $22.2 \%$ & $21.4 \%$ & 0.488 \\
\hline Atrial fibrillation & $6.5 \%$ & $5.4 \%$ & $6.2 \%$ & $8.7 \%$ & 0.082 \\
\hline $\mathrm{CHF}$ & $11.2 \%$ & $7.3 \%$ & $8.7 \%$ & $15.9 \%$ & $<0.001$ \\
\hline Last pre-op serum creatinine (mean, SD) & $1.1 \pm 0.6$ & $1.1 \pm 0.5$ & $1.1 \pm 1.4$ & $1.3 \pm 1.0$ & 0.464 \\
\hline Diabetes & $38.0 \%$ & $34.6 \%$ & $33.9 \%$ & $43.8 \%$ & 0.001 \\
\hline Ejection fraction $<40 \%$ & $12.1 \%$ & $10.6 \%$ & $10.0 \%$ & $12.3 \%$ & 0.508 \\
\hline Hypertension & $81.0 \%$ & $79.2 \%$ & $80.1 \%$ & $83.0 \%$ & 0.273 \\
\hline IABP pre-op & $3.8 \%$ & $4.6 \%$ & $4.8 \%$ & $2.7 \%$ & 0.165 \\
\hline \multicolumn{6}{|l|}{ Prior Ml } \\
\hline No & $54.6 \%$ & $57.3 \%$ & $57.4 \%$ & $53.7 \%$ & \multirow[t]{5}{*}{0.098} \\
\hline$<24$ h pre-op & $1.5 \%$ & $1.4 \%$ & $1.5 \%$ & $2.1 \%$ & \\
\hline$>24 \mathrm{~h} \&<7$ days pre-op & $20.5 \%$ & $18.7 \%$ & $19.6 \%$ & $18.0 \%$ & \\
\hline$>7$ days $\&<365$ days pre-op & $9.8 \%$ & $7.9 \%$ & $8.45 \%$ & $13.6 \%$ & \\
\hline$>365$ days pre-op & $13.6 \%$ & $14.7 \%$ & $12.7 \%$ & $12.6 \%$ & \\
\hline Vascular disease & $27.8 \%$ & $26.1 \%$ & $25.2 \%$ & $30.2 \%$ & 0.154 \\
\hline Unstable angina & $58.2 \%$ & $53.7 \%$ & $55.0 \%$ & $58.1 \%$ & 0.347 \\
\hline COPD & $12.6 \%$ & $11.2 \%$ & $12.1 \%$ & $15.5 \%$ & 0.095 \\
\hline Left main, $\geq 50 \%$ stenosis & $31.5 \%$ & $33.0 \%$ & $35.3 \%$ & $33.0 \%$ & 0.669 \\
\hline Prior CABG & $2.4 \%$ & $2.3 \%$ & $2.8 \%$ & $1.8 \%$ & 0.566 \\
\hline Prior PCl & $19.6 \%$ & $20.5 \%$ & $18.7 \%$ & $20.0 \%$ & 0.760 \\
\hline \multicolumn{6}{|l|}{ Priority } \\
\hline Emergent & $1.5 \%$ & $2.5 \%$ & $1.7 \%$ & $1.6 \%$ & \multirow[t]{3}{*}{0.815} \\
\hline Urgent & $70.1 \%$ & $68.0 \%$ & $67.4 \%$ & $67.8 \%$ & \\
\hline Non-urgent & $28.3 \%$ & $29.5 \%$ & $30.8 \%$ & $30.6 \%$ & \\
\hline Received pRBC units & & $30.4 \%$ & $35.5 \%$ & $48.6 \%$ & $<0.001$ \\
\hline \multicolumn{6}{|l|}{ Number of pRBC units given pre-op } \\
\hline 0 & $97.9 \%$ & $99.4 \%$ & $97.5 \%$ & $97.1 \%$ & \multirow[t]{2}{*}{0.142} \\
\hline 1 or more & $2.1 \%$ & $0.6 \%$ & $2.5 \%$ & $2.9 \%$ & \\
\hline
\end{tabular}

a(Mean, SD)

$A K I$ acute kidney injury, KDIGO Kidney Disease: Improving Global Outcomes, $B M I$, body mass index $\left(\mathrm{kg} / \mathrm{m}^{2}\right), B S A$ body surface area $\left(\mathrm{m}^{2}\right), C A B G$ coronary artery bypass graft, $C H F$ congestive heart failure, COPD chronic obstructive pulmonary disease, IABP intra-operative balloon pump, $M I$ myocardial infarction, $P C I$ percutaneous coronary intervention, $R B C$ red blood cell, $S D$ standard deviation

In the kidney, Gal-3 has multiple functions including regulating the inflammatory response and cell growth, proliferation, and differentiation [24, 25]. Gal-3 has been proposed to be a marker of combined cardiac and renal fibrosis in the chronic setting [24, 25]. The prognostic value of baseline impaired cardiac and renal functional reserve may predict risk of AKI after cardiac surgery [26]. A 15\% change from baseline has been associated with significantly more heart failure hospitalizations and 
Table 3 Unadjusted and STS adjusted model evaluating preoperative Gal-3 measurements and association with KDIGO stage severity

\begin{tabular}{|c|c|c|c|c|c|c|}
\hline & \multicolumn{3}{|c|}{ KDIGO Stage 1} & \multicolumn{3}{|c|}{ KDIGO Stage 2 or 3} \\
\hline & $\mathrm{OR}$ & $95 \% \mathrm{Cl}$ & $p$ value & $\mathrm{OR}$ & $95 \% \mathrm{Cl}$ & $p$ value \\
\hline \multicolumn{7}{|l|}{ Unadjusted } \\
\hline Preoperative & 1.03 & $1.01-1.04$ & 0.000 & 1.04 & $1.02-1.06$ & 0.000 \\
\hline Natural log & 1.47 & $1.19-1.81$ & 0.000 & 2.03 & $1.40-2.94$ & 0.000 \\
\hline \multicolumn{7}{|l|}{ Tertiles } \\
\hline 1 & 1.00 & $1.00-1.00$ & & 1.00 & $1.00-1.00$ & \\
\hline 2 & 1.12 & $0.83-1.50$ & 0.456 & 1.36 & $0.74-2.52$ & 0.322 \\
\hline 3 & 1.70 & $1.28-2.27$ & 0.000 & 2.86 & $1.63-5.01$ & 0.000 \\
\hline Preoperative above median & 1.36 & $1.08-1.72$ & 0.009 & 2.44 & $1.53-3.89$ & 0.000 \\
\hline \multicolumn{7}{|c|}{ STS Readmission Prediction Model ${ }^{a}$} \\
\hline Preoperative & 1.03 & $1.01-1.04$ & 0.002 & 1.03 & $1.00-1.06$ & 0.005 \\
\hline Natural log & 1.40 & $1.10-1.78$ & 0.006 & 1.87 & $1.23-2.86$ & 0.004 \\
\hline \multicolumn{7}{|l|}{ Tertiles } \\
\hline 1 & 1.00 & $1.00-1.00$ & & 1.00 & $1.00-1.00$ & \\
\hline 2 & 1.08 & $0.79-1.48$ & 0.625 & 1.37 & $0.73-2.56$ & 0.329 \\
\hline 3 & 1.71 & $1.24-2.37$ & 0.001 & 2.95 & $1.63-5.34$ & 0.000 \\
\hline Preoperative above median & 1.30 & $1.00-1.68$ & 0.046 & 2.31 & $1.39-3.85$ & 0.001 \\
\hline \multicolumn{7}{|c|}{ STS Readmission Prediction Model + NT-pro BNP } \\
\hline Preoperative & 1.02 & $1.01-1.04$ & 0.005 & 1.03 & $1.00-1.06$ & 0.007 \\
\hline Natural log & 1.31 & $1.03-1.68$ & 0.028 & 1.73 & $1.13-2.65$ & 0.012 \\
\hline \multicolumn{7}{|l|}{ Tertiles } \\
\hline 1 & 1.00 & $1.00-1.00$ & & 1.00 & $1.00-1.00$ & \\
\hline 2 & 1.08 & $0.79-1.48$ & 0.631 & 1.36 & $0.72-2.57$ & 0.337 \\
\hline 3 & 1.65 & $1.19-2.29$ & 0.003 & 2.85 & $1.57-5.16$ & 0.001 \\
\hline Preoperative above median & 1.28 & $0.99-1.66$ & 0.060 & 2.26 & $1.35-3.76$ & 0.002 \\
\hline
\end{tabular}

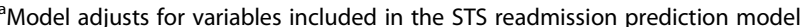

KDIGO Kidney Disease: Improving Global Outcomes, Cl confidence interval, OR odds ratio, STS Society of Thoracic Surgeons

increased mortality compared with lower and decreasing levels [21]. In addition, Gal-3 has been shown to stimulate macrophages to release pro-inflammatory cytokines (e.g. MCP-1, IL-6, and IL-1B) and produce reactive oxygen species, enhancing the inflammatory response in the kidney [17]. Knocking-out the Gal-3 gene or directly

Table 4 Model comparison statistics evaluating the discriminatory power of the base regression model and the additive value of preoperative Gal-3 terciles and preoperative NT-proBNP terciles

\begin{tabular}{llc}
\hline & C-statistic (95\% Cl) & $\begin{array}{l}\text { ROCCOMP } \\
p \text { value }\end{array}$ \\
\hline STS Readmission Prediction Model & $0.69(0.66-0.71)$ & \\
STS model + Gal-3 preoperative terciles & $0.70(0.67-0.72)$ & 0.042 \\
STS model + combined Gal-3 and & $0.70(0.68-0.73)$ & 0.005 \\
NT-pro BNP preoperative terciles & & \\
\hline${ }^{a}$ ROC comparison against base model & &
\end{tabular}

inhibiting the Gal-3 protein is known to inhibit renal fibrosis and lessen renal injury in AKI [16, 27, 28].

We have demonstrated that preoperative levels of circulating Gal-3 are associated with AKI and AKI severity after cardiac surgery. Patients with higher levels of circulating Gal-3 may be predisposed to excessive inflammatory processes. Preoperative Gal-3 levels could also be acting as a marker for early CKD, identifying patients more susceptible to AKI because of underlying kidney disease. Further, preoperative Gal-3 may be serving as an indicator of heart failure (HF) and those patients at risk of AKI due to ischemic renal injury secondary to pump failure. Preoperative measurement of Gal-3 may provide a means to evaluate AKI risk due to multiple etiologies.

Multiple preoperative biomarkers have been evaluated for their ability to predict AKI after cardiac surgery. Cystatin $\mathrm{C}(\mathrm{CysC})$ is a circulating protease inhibitor and correlates with the glomerular filtration 
Table 5 Model evaluation Gal3 \& eGFR

\begin{tabular}{|c|c|c|c|c|c|c|}
\hline & \multicolumn{6}{|c|}{ STS Readmission Prediction Model + Risk Marker } \\
\hline & C-statistic (95\% Cl) & NRI & NRI $P$ & $|\mathrm{D}|$ & $\mid \mathrm{DI} P$ & Test of Equality $P^{b}$ \\
\hline STS Readmission Model & $0.69(0.66-0.71)$ & & & & & \\
\hline Preoperative Gal-3 terciles & $0.70(0.67-0.72)$ & 0.03 & 0.067 & 0.01 & 0.000 & 0.042 \\
\hline Preoperative eGFR ${ }^{a}(\mathrm{~mL} / \mathrm{min} / 1.73 \mathrm{~m} \wedge 2)$ & $0.69(0.66-0.72)$ & 0.02 & 0.124 & 0.00 & 0.010 & 0.302 \\
\hline
\end{tabular}

$P$ represents the statistical $p$ value.

${ }^{a}$ Estimated glomerular filtration rate (eGFR)

${ }^{\mathrm{b}} \mathrm{ROC}$ comparison against base model

NRI Net Reclassification Improvement index, IDI Integrated Discrimination Improvement index

rate (GFR) [13]. Its preoperative values have been shown to independently associate with AKI following cardiac surgery $[29,30]$. Similarly, prior research has demonstrated a relationship between brain natriuretic peptide (BNP), NT-proBNP and Gal-3 with elevation in both markers related to outcomes [31]. Preoperative BNP, a polypeptide released by the ventricles in response to volume overload, has been shown to predict postoperative development of AKI [29, 32]. The inclusion of NT-proBNP in our study resulted in only a moderate difference from our adjusted prediction model. Compared to the adjusted model, the above median preoperative Gal3 measurements were non-signfiicant for those developing KDIGO Stage 1 in augmented model with NT-proBNP. The inclusion of BNP in our prediction model could provide important inferences on cardiac-surgery associated with AKI and heart failure, but further evaluation is needed. Given the varying kinetics and characteristics of individual biomarkers, it is likely that the measurement of multiple biomarkers, in addition to Gal-3, is necessary to accurately perform preoperative risk assessment for AKI $[29,33]$.

The primary strength of this study is its large sample size comprised of patients who underwent cardiac surgery at eight hospitals across Maine, Vermont and New Hampshire. Studies on preoperative biomarker levels and prediction of AKI have been previously conducted with small cohorts, in single-center settings and lacked defined, explicit outcomes [34]. In this study, we leveraged the NNECDSG registry, which is comprehensive in the patient and procedural data that it contains. The completeness and quality of this data also helps ensure that adequate adjustment was carried out.

\section{Study limitations}

There are limitations to this study to consider. First, we lacked detailed information on some conditions known to affect the incidence of AKI including cardiopulmonary bypass times, hemodynamics or the use of inotropic and vasoactive drugs in the perioperative period. Therefore, residual confounding of the demonstrated association of Gal-3 with AKI can not be excluded. Gal-3 has been found to be correlated with pre-existing renal disease and heart failure. Medical support to maintain blood pressure arguably would be more aggressive in patients with higher Gal-3 values, and our results would more likely be biased towards the null-hypothesis. Secondly, we also used creatinine-based definitions for AKI which are relatively insensitive and non-specific in the period directly following insults to the kidney [35]. Thirdly, we were also unable to evaluate long-term outcomes such as major adverse renal and cardiac events (MARCE). Fourthly, given the unique patient characteristics associated with the CABG patient population, there may be reproducibility limitations with a more heterogeneous population. Finally, the mean imputation method used to address missing data may influence the overall composition and performance of the prediction model.

\section{Conclusion}

Improving the predictive ability of AKI risk prior to surgery is critical to take preventative measures and counsel patients on potential outcomes after cardiac surgery. Elevated preoperative Gal-3 levels may be used to augment risk information for patients at greatest risk of developing AKI and AKI severity after cardiac surgery. If Gal-3 is elevated, there are several AKI mitigation strategies to employ including avoiding surgery on the same day as cardiac catherization, limiting transfusion, remote ischemic preconditioning prior to surgery and stopping angiotensin-converting enzymes inhibitors (ACEIs) and angiotensin receptor blockers (ARBs) for 2 days after surgery.

We are the first to demonstrate a significant association between the inflammatory and fibrosis biomarker Gal-3 and AKI in patients undergoing cardiac surgery. Our findings suggest that preoperative Gal-3 levels could be used to identify patients at the highest risk of developing AKI after cardiac surgery. 


\section{Appendix}

Table 6 STS Model Variables and NNE Registry Data

\begin{tabular}{|c|c|}
\hline STS & NNE \\
\hline \multicolumn{2}{|c|}{$\begin{array}{l}\text { 1. We were unable to adjust for chronic lung disease or prior myocardial infarction in the same way as the investigato } \\
\text { preoperative readmission model. }\end{array}$} \\
\hline $\begin{array}{l}\text { STS registry had data on the severity of } \\
\text { chronic lung disease (none, mild, moderate, } \\
\text { severe) }\end{array}$ & $\begin{array}{l}\text { NNE registry only contains information on w } \\
\text { or not members of our patient cohort had c } \\
\text { obstructive pulmonary disease (COPD) or no }\end{array}$ \\
\hline \multicolumn{2}{|c|}{ 2. The STS and NNE registries also categorize prior myocardial infarctions in different ways. } \\
\hline $\begin{array}{l}\text { STS uses four different categories } \\
\text { (no recent Ml, MI between one and } \\
21 \text { days ago, MI more than six and less } \\
\text { than } 24 \mathrm{~h} \text { ago, and Ml less than or equal } \\
\text { to } 6 \mathrm{~h} \text { ago) }\end{array}$ & $\begin{array}{l}\text { NNE registry instead uses five categories for } \\
\text { our cohort (no prior Ml, Ml less than } 24 \mathrm{~h} \\
\text { prior to operation, MI more than } 24 \mathrm{~h} \text { but } \\
\text { less than } 7 \text { days prior to operation, Ml more } \\
\text { than } 7 \text { days but less than } 1 \text { year prior to } \\
\text { operation, and Ml more than } 0 \text { year prior to } \\
\text { operation) }\end{array}$ \\
\hline
\end{tabular}

3. We were unable to adjust for immunosuppressive treatment at all, since the NNE registry did not collect that information for our cohort.

4. Our final NNE version of the STS preoperative readmission risk adjustment model included 30 covariates.

This table describes the differences in variables between the STS model and variables available in the NNE registry dataset

\section{Abbreviations}

ACEls: Angiotensin-converting enzymes inhibitors; AKl: Acute kidney injury; AKIN: Acute Kidney Injury Network; AMI: Acute myocardial infarction; ARB: Angiotensin receptor blockers; BMI: Body mass index; BSA: Body surface area; CABG: Coronary artery bypass graft; CHF: Chronic heart failure; Cl: Confidence interval; COPD: Chronic obstructive pulmonary disease; IABP: Intra-aortic balloon pump; IL-1: Interleukin-1; KDIGO: Kidney Disease: Improving Global Outcomes; MARCE: Major adverse renal and cardiac events; MI: Myocardial infarction; NNE: Northern New England; NNECDSG: Northern New England Cardiovascular Disease Study Group; OR: Odds ratio; PCI: Percutaneous coronary intervention; PRBC: Packed red blood cells; ST2: Serum soluble ST2; STS: Society of Thoracic Surgeons; VAD: Ventricular assist device

\section{Acknowledgements}

Not applicable.

\section{Funding}

This research is supported by the National Heart Lung and Blood Institute R01HL119664 (PI: Brown). All authors are research staff or investigators on the grant.

\section{Availability of data and materials}

The datasets used and/or analysed during the current study are available from the corresponding author on request.

\section{Authors' contributions}

$M W B, D L, M R$, and $K L$ made substantial contributions to conception and design and analysis and interpretation of the data. SO, HTP and TA was involved in the drafting and revising and critical to the analysis and interpretation of the data. SA and DP made substantial contributions to conception and design, was involved in the drafting and revising it critically for important intellectual content. JR made significant contributions to conception and design, was involved in the drafting and revising, acquired the data, and was critical in the analysis and interpretation of the data. All authors read and approved the final manuscript.

\section{Ethics approval and consent to participate}

The Committee for the Protection of Human Subjects at Dartmouth College (Institutional Review Board) approved this study for both the prospective cohort with patient consent and the linkage of readmission and mortality events.

\section{Consent for publication}

Not applicable.

\section{Competing interests}

The authors declare that they have no competing interests.

\section{Publisher's Note}

Springer Nature remains neutral with regard to jurisdictional claims in published maps and institutional affiliations.

\section{Author details}

${ }^{1}$ Division of Cardiovascular and Thoracic Surgery, Duke University Medical Center, Durham, NC, USA. ${ }^{2}$ Institute for Healthcare Policy and Innovation, University of Michigan, Ann Arbor, MI, USA. ${ }^{3}$ Section of Health Services Research and Quality, Department of Cardiac Surgery, University of Michigan, Ann Arbor, MI, USA. ${ }^{4}$ Section of Urology, Department of Surgery, Dartmouth-Hitchcock Medical Center, Lebanon, $\mathrm{NH}$, USA. ${ }^{5}$ Carolinas HealthCare System, Charlotte, NC, USA. ${ }^{6}$ The Dartmouth Institute for Health Policy and Clinical Practice, Geisel School of Medicine, Lebanon, NH, USA.

${ }^{7}$ Division of Nephrology, Department of Medicine, Johns Hopkins University, Baltimore, MD, USA. ${ }^{8}$ Department of Biomedical Data Science, HB 7505 Dartmouth-Hitchcock Medical Center, Lebanon, NH NH 03756, USA.

${ }^{9}$ Department of Epidemiology, Geisel School of Medicine, Lebanon, NH, USA.

Received: 9 March 2018 Accepted: 9 October 2018

Published online: 20 October 2018

\section{References}

1. Schaub JA, Parikh CR. Biomarkers of acute kidney injury and associations with short- and long-term outcomes. F1000Res. 2016;5.

2. Rosner MH, Okusa MD. Acute kidney injury associated with cardiac surgery. Clin J Am Soc Nephrol. 2006;1 (1):19-32.

3. O'Neal JB, Shaw AD, Billings FT. Acute kidney injury following cardiac surgery: current understanding and future directions. Crit Care. 2016;20(1): 187.

4. Brown JR, Malenka DJ, DeVries JT, Robb JF, Jayne JE, Friedman BJ, Hettleman BD, Niles NW, Kaplan AV, Schoolwerth AC, Thompson CA. Transient and persistent renal dysfunction are predictors of survival after percutaneous coronary intervention: insights from the Dartmouth Dynamic Registry. Catheter Cardiovasc Interv. 2008;72(3):347-54.

5. Brown JR, Parikh CR, Ross CS, et al. Impact of perioperative acute kidney injury as a severity index for thirty-day readmission after cardiac surgery. Ann Thorac Surg. 2014:97(1):111-7.

6. Goldenberg I, Chonchol M, Guetta V. Reversible acute kidney injury following contrast exposure and the risk of long-term mortality. Am J Nephrol. 2009;29(2):136-44. 
7. Thakar CV, Parikh PJ, Liu Y. Acute kidney injury (AKI) and risk of readmissions in patients with heart failure. Am J Cardiol. 2012;109(10):1482-6.

8. Vaidya VS, Ferguson MA, Bonventre JV. Biomarkers of acute kidney injury. Annu Rev Pharmacol Toxicol. 2008:48:463-93.

9. Drechsler C, Delgado G, Wanner C, et al. Galectin-3, renal function, and clinical outcomes: results from the LURIC and 4D studies. J Am Soc Nephrol. 2015;26(9):2213-21.

10. Ho JE, Liu C, Lyass A, et al. Galectin-3, a marker of cardiac fibrosis, predicts incident heart failure in the community. J Am Coll Cardiol. 2012;60(14):1249-56.

11. O'Seaghdha CM, Hwang SJ, Ho JE, Vasan RS, Levy D, Fox CS. Elevated galectin-3 precedes the development of CKD. J Am Soc Nephrol. 2013;24(9): 1470-7.

12. Parikh CR, Coca SG, Thiessen-Philbrook H, et al. Postoperative biomarkers predict acute kidney injury and poor outcomes after adult cardiac surgery. $J$ Am Soc Nephrol. 2011;22(9):1748-57.

13. Dharnidharka VR, Kwon C, Stevens G. Serum cystatin C is superior to serum creatinine as a marker of kidney function: a meta-analysis. Am J Kidney Dis. 2002;40(2):221-6.

14. Nishiyama J, Kobayashi S, Ishida A, et al. Up-regulation of galectin-3 in acute renal failure of the rat. Am J Pathol. 2000;157(3):815-23.

15. Henderson NC, Mackinnon AC, Farnworth SL, et al. Galectin-3 expression and secretion links macrophages to the promotion of renal fibrosis. Am J Pathol. 2008:172(2):288-98.

16. Kolatsi-Joannou M, Price KL, Winyard PJ, Long DA. Modified Citrus pectin reduces Galectin-3 expression and disease severity in experimental acute kidney injury. PLoS One. 2011;6(4):e18683.

17. Fernandes Bertocchi AP, Campanhole G, Wang PH, et al. A role for galectin3 in renal tissue damage triggered by ischemia and reperfusion injury. Transplant international : official journal of the European Society for Organ Transplantation. 2008;21(10):999-1007.

18. Ji $F$, Zhang $S$, Jiang $X$, et al. Diagnostic and prognostic value of galectin-3, serum creatinine, and cystatin C in chronic kidney diseases. Journal of clinical laboratory analysis. 2017;31:5.

19. Kidney Disease. Improving Global Outcomes (KDIGO) Acute Kidney Injury Work Group. KDIGO Clinical Practice Guideline for Acute Kidney Injury. Kidney inter, Suppl. 2012;2:1-138.

20. Shahian DM, He X, O'Brien SM, Grover FL, Jacobs JP, Edwards FH, Welke KF, Suter LG, Drye E, Shewan CM, Han L, Peterson E. Development of a clinical registry-based 30-day readmission measure for coronary artery bypass grafting surgery. Circulation. 2014;130(5):399-409.

21. de Boer RA, Yu L, van Veldhuisen DJ. Galectin-3 in cardiac remodeling and heart failure. Current Heart Failure Reports. 2010;7(1):1-8.

22. de Boer RA, van Veldhuisen DJ, Gansevoort RT, et al. The fibrosis marker galectin-3 and outcome in the general population. J Intern Med. 2012; 272(1):55-64.

23. Shah RV, Chen-Tournoux AA, Picard MH, van Kimmenade RRJ, Januzzi JL. Galectin-3, cardiac structure and function, and long-term mortality in patients with acutely decompensated heart failure. Eur J Heart Fail. 2010; 12(8):826-32.

24. Chen SC, Kuo PL. The role of Galectin-3 in the kidneys. Int J Mol Sci. 2016; 17(4):565.

25. Desmedt V, Desmedt S, Delanghe JR, Speeckaert R, Speeckaert MM. Galectin-3 in renal pathology: more than just an innocent bystander. Am J Nephrol. 2016;43(5):305-17.

26. Hundae A, McCullough PA. Cardiac and renal fibrosis in chronic cardiorenal syndromes. Nephron Clin Pract. 2014;127(1-4):106-12.

27. Varrier M, Forni LG, Ostermann M. Long-term sequelae from acute kidney injury: potential mechanisms for the observed poor renal outcomes. Crit Care. 2015;19(1):102.

28. Calvier L, Martinez-Martinez E, Miana M, et al. The impact of galectin-3 inhibition on aldosterone-induced cardiac and renal injuries. JACC Heart failure. 2015;3(1):59-67.

29. Koyner JL, Parikh CR. Clinical utility of biomarkers of AKI in cardiac surgery and critical illness. Clin J Am Soc Nephrol. 2013;8(6):1034-42.

30. Shlipak MG, Coca SG, Wang Z, et al. Presurgical serum cystatin $C$ and risk of acute kidney injury after cardiac surgery. American journal of kidney diseases : the official journal of the National Kidney Foundation. 2011;58(3): $366-73$

31. McCullough PA, Olobatoke A, Vanhecke TE. Galectin-3: a novel blood test for the evaluation and management of patients with heart failure. Rev Cardiovasc Med. 2011;12(4):200-10.
32. Patel UD, Garg AX, Krumholz HM, et al. Preoperative serum brain natriuretic peptide and risk of acute kidney injury after cardiac surgery. Circulation. 2012;125(11):1347-55.

33. de Geus HR, Betjes MG, Bakker J. Biomarkers for the prediction of acute kidney injury: a narrative review on current status and future challenges. Clin Kidney J. 2012;5(2):102-8.

34. O'Brien SM, Clarke DR, Jacobs JP, et al. An empirically based tool for analyzing mortality associated with congenital heart surgery. J Thorac Cardiovasc Surg. 2009:138(5):1139-53.

35. Moran SM, Myers BD. Course of acute renal failure studied by a model of creatinine kinetics. Kidney Int. 1985;27(6):928-37.

\section{Ready to submit your research? Choose BMC and benefit from:}

- fast, convenient online submission

- thorough peer review by experienced researchers in your field

- rapid publication on acceptance

- support for research data, including large and complex data types

- gold Open Access which fosters wider collaboration and increased citations

- maximum visibility for your research: over $100 \mathrm{M}$ website views per year

At BMC, research is always in progress.

Learn more biomedcentral.com/submissions 